\title{
Bowler analysis in cricket using centre of mass inertial monitoring
}

David Rowlands ${ }^{1, \star}$, Daniel Arthur James ${ }^{1,2}$ and David Thiel ${ }^{1}$

${ }_{1}$ Centre for Wireless Monitoring and Applications, Griffith University, Australia

${ }_{2}$ Centre of Excellence for Sport Science Research, Queensland Academy of Sport, Australia 


\section{Introduction:}

Inertial sensors (accelerometers and gyroscopes) are now commonly used in human monitoring for energy output [1, 2], athlete monitoring [2-8] and disease assessment [9]. Small, battery powered, portable units can be used to log or relay data via a wireless link to a computer for analysis and assessment. This allows monitoring the subject in their natural environment without space restrictions and unencumbered by a wired connection. In sports monitoring other commonly used technologies include multi-camera systems for automatic tracking [9], video recording $[9,10]$ and training machines such as treadmills, stationary cycling machines and stationary rowing machines.

The location of miniature inertial sensors in a small, battery powered unit at the sacrum is as close as possible to the centre of mass (CoM). Work previously reported using CoM monitoring for athlete assessment includes running [2], swimming [3,4], and snow boarding [5,6]. There are many advantages in using this position on the human body, including:

o Unrestricted movement of the athlete

o Minimal detectable effect on athlete performance both psychologically and physically

o Minimal effect on athlete balance

Recently there has been significant interest in monitoring the biomechanics of bowlers in cricket [11-16]. The main objectives of this work have been in injury prevention, assessment and recovery, and in the assessment of technique for the development of sub-elite athletes for further training to elite level. There are three accepted classifications of fast bowling action, although there is a continuum between the three styles [13]: 
Side-on - the bowler turns side on one or two strides before releasing the ball

Front-on - the bowler maintains a face-on position during the ball release

Mixed - a combination of side-on and front-on.

Previous researchers have measured the following parameters as part of bowling assessment.

(a) Peak speed (run-up) [12, 13]

(b) Pre-delivery stride length [13]

(c) Ground reaction force back foot during ball release [13, 15]

(d) Ground reaction force front foot during ball release [13, 15]

(e) Shoulder and hip rotation $[11,13,15]$

This preliminary paper reports the use of miniature inertial sensors located at the sacrum for bowling assessment during a front-on bowling action. Some of the parameters listed above can be extracted from this data.

\section{Methods and Experimental:}

In this paper previously developed instrumentation was used to monitor bowler action [2,3,4]. The sensors consisted of a tri-axial accelerometer and gyroscopes with ranges of $+/-10 \mathrm{~g}$, and $250 \%$ s respectively. The data was sampled at $200 \mathrm{~Hz}$ and transmitted using a class 1 Bluetooth link to a PC. Data display and acquisition was handled using a purpose built Visual Basic client with subsequent analysis in Matlab. The sensors were calibrated using static orientations. The sensors were placed on the sacrum as a reasonable approximation of the CoM with minimal skin and muscle artefact. Figure 1 shows the bowler close to delivery. The arrow shown on the figure indicates the primary acceleration axis and rotational component. 
Several bowlers were used in the study with a representative case presented (left hand delivery). The protocol involved a warm-up period prior to the collection of data. The Bowlers were instructed to use a set run up length and to bowl at a sub maximal pace to a facing batsman in practice nets, a typical training environment. Video footage was also collected to aid in event detection and verification.

\section{Results:}

Data from the sensor unit was measured for each bowling action and was matched to the video data. The main bowling events that are apparent from the video are:

- The run up over a short distance.

- The back foot strike (B) prior to delivery and subsequent counter-clockwise rotation of the hips.

- Front foot strike (F) and clock-wise rotation of the hips, which continues through to ball release.

- Follow through after ball release.

Figure 2 shows data from a sample delivery and shows acceleration and rate gyroscope data with respect to the vertical axis. Figure 2(a) shows the acceleration in the vertical axis with positive being the upwards direction and negative being the downwards direction. Figure 2(b) shows the angular velocity around the vertical axis with a counter clockwise rotation indicated by a positive direction and clockwise rotation indicated by a negative direction. 
Bowling action events are clearly evident in figure 2 with the run up preceding hip rotation and delivery. The run up consists of 5 steps before the back foot strike (B). When the back foot strike occurs, the bowler starts to rotate the hips in a counter-clockwise direction before the front foot strike (F). When the front foot strike occurs the bowler starts to rotate the hips in a clock-wise direction. While ball release is not apparent in either of the accelerometer or gyroscope data additional sensors could be used in future. The order and timing of the bowling action matches both the video and the expected bowling order [13], which indicates that the inertial sensors placed at the CoM can pick up the order of the important events in the bowling action.

Total hip rotation was determined using a short-term cumulative trapezoidal integration of the angular velocity after appropriate baseline removal. Hip rotation was found to be 40 degrees of rotation in the counter-clockwise direction followed by a rotation of about 30 degrees in the clockwise direction. It is assumed that the difference in the rotation is due to the angle of foot and leg after the ball has been released. In the video, the bowler appeared to step across the body.

Figure 3 shows the step rate, speed, and step length derived from ground contact events and period in the accelerometer data [2]. The bar marked with a B indicates the Back foot strike and the bar marked with an F indicates the front foot strike. The first step, usually a hop, in the run up is omitted since the technique is not valid for this motion. This technique was developed from steady state running by Wixted [2] and is a useful first order approximation for analysis of the cricket run-up even though the bowling run up is very short with only a few steps. 
From Figure 3, it is apparent that the bowler starts the run up with shorter steps at a high step rate giving a low speed and ends the run up with a higher step rate and larger step length giving a higher overall speed. It can also be seen that the speed is consistent a few paces prior to delivery which is reported in the literature [13]. The peak run up speed can be seen as about $2.7 \mathrm{~m} / \mathrm{s}$ with a predelivery stride of about $1.2 \mathrm{~m}$ which is consistent with the literature.

\section{Conclusions and Further work:}

This paper has demonstrated that one can extract useful parameters from the CoM that quantify the action of a bowler. These parameters include the timing of the important events in the bowling action, number of steps, step rate, peak run-up speed, step length and hip rotation with very little signal processing. Further analysis of the data should allow an assessment of the smoothness of the bowling action through the crease, the possible problems which might occur after many repetitions based on hip rotation and the difference between fast bowling around or over the wicket. Further work will include a study to determine the ground reaction forces, and a study correlating the ball speed to the bowling parameters. The extraction of quantitative data with relative ease allows those in cricket to track the performance and action of an athlete longitudinally. This assessment allows comparison between athletes, monitoring of the efficacy of training regimes and aids in injury prevention through changes over time, post recovery and with fatigue.

Acknowledgements: The authors wish to thank Neeli Madhusudan Rao and Andrew Busch for their assistance in this research. 


\section{References:}

1. Wong, T.C., Webster, J.G., Montoye, H.J., Washburn, R., Portable Accelerometer Device for Measuring Human Energy Expenditure, IEEE Trans. Biomedical Engineering, 1981; BME-28 (6), $467-471$

2. Wixted, A.J., Thiel, D.V., Hahn, A. G., Gore, C. J., Pyne, D. B., James, D.A., Measurement of energy expenditure in elite athletes using MEMS-based triaxial accelerometers. IEEE Sensors Journal, 2007; 7(4), 481-488.

3. Davey, N.P., Anderson, M.E., James, D.A., An accelerometer-based system for elite athlete swimming performance analysis. Proc. SPIE, 2005; 5649, 409 (2005);

DOI:10.1117/12.582264

4. Neil Davey, Megan Anderson and Daniel A. James. Validation trial of an accelerometerbased sensor platform for swimming. Sports Technology 2008, 1, No. 4-5, 202-207, DOI: $10.1002 / j s t .59$

5. Ahmadi, A., Rowlands, D.D., James, D.A., Investigating the translational and rotational motion of the swing using accelerometers for athlete skill assessment. $5^{\text {th }}$ IEEE Conf. Sensors 2006; 980-983.

6. Harding, J.W., Small, J.W., and James, D.A., Feature extraction of performance variables in elite half-pipe snowboarding using body mounted inertial sensors. Proc. SPIE. 2007; 6799, 679917; DOI:10.1117/12.759259

7. Jason William Harding, Colin Gordon Mackintosh, David Thomas Martin, Allan Geoffrey Hahn and Daniel Arthur James. Automated scoring for elite half-pipe snowboard competition: important sporting development or techno distraction? Sports Technology 2008, 1, No. 6, in press, DOI: 10.1002/jst.69 
8. Matthew Brodie, Alan Walmsley and Wyatt Page. Fusion motion capture: a prototype system using inertial measurement units and GPS for the biomechanical analysis of ski racing. Sports Technology 2008, 1, No. 1, 17-28, DOI: 10.1002/jst.6

9. Van Someren, E.J.W., Actigraphic monitoring of movement and rest-activity rhythms in aging, Alzheimer's disease, and Parkinson's disease. IEEE Trans. Rehabilitation Engineering 1997; 5(4), 394-398.

10. Liebermann, D.G., Katz, L., Hughes, M.D.,Bartlett, R.M., McClements, J., Franks, I.M., Advances in the application of information technology to sport performance. J. Sports Sci. 2002; 20, 755-769.

11. Portus, M.R., Sinclair, P.J., Burke, S.T., Moore, D.J.A., Farhart, P.J., Cricket fast bowling and technique and the influence of selected physical factors during an 8-over spell. J. Sports Sci. 2000; 18, 999-1011.

12. Pyne, D.B., Duthie, G.M., Saunders, P.U., Petersen, C.A., Portus, M.R., Anthropometric and strength correlates of fast bowling speed in junior and senior cricketers. J. Strength \& Conditioning Res. 2006; 20(3), 620-626.

13. Bartlett, R.M., Stickill, N.P., Elliot, B.C., Burnett, A.F., The biomechanics of fast bowling in men's cricket: A review. J. Sports Sci. 1996; 14, 403-424.

14. Dennis, R.J., Finch, C.F., Farhart, P.J., Is bowling workload a risk factor for injury to Australia junior crocket fast bowlers? Br. J. Sports. Med. 2005; 39, 843-846.

15. Elliott, B.C., Back injuries and the fast bowler in cricket. J. Sports Sci. 2000; 18, 931991.

16. Kibbler, W.B., The role of the scapula in athletic shoulder function. Am. J. Sports Med. 1998; 26(2), 325-337. 


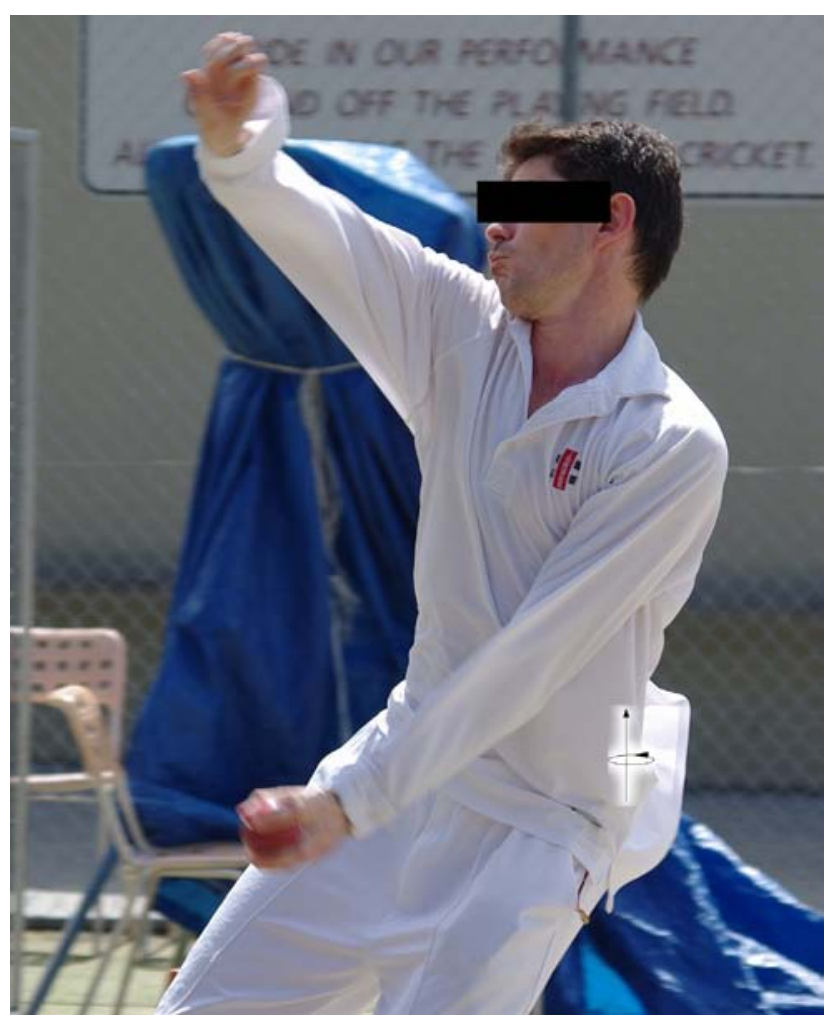

Figure 1
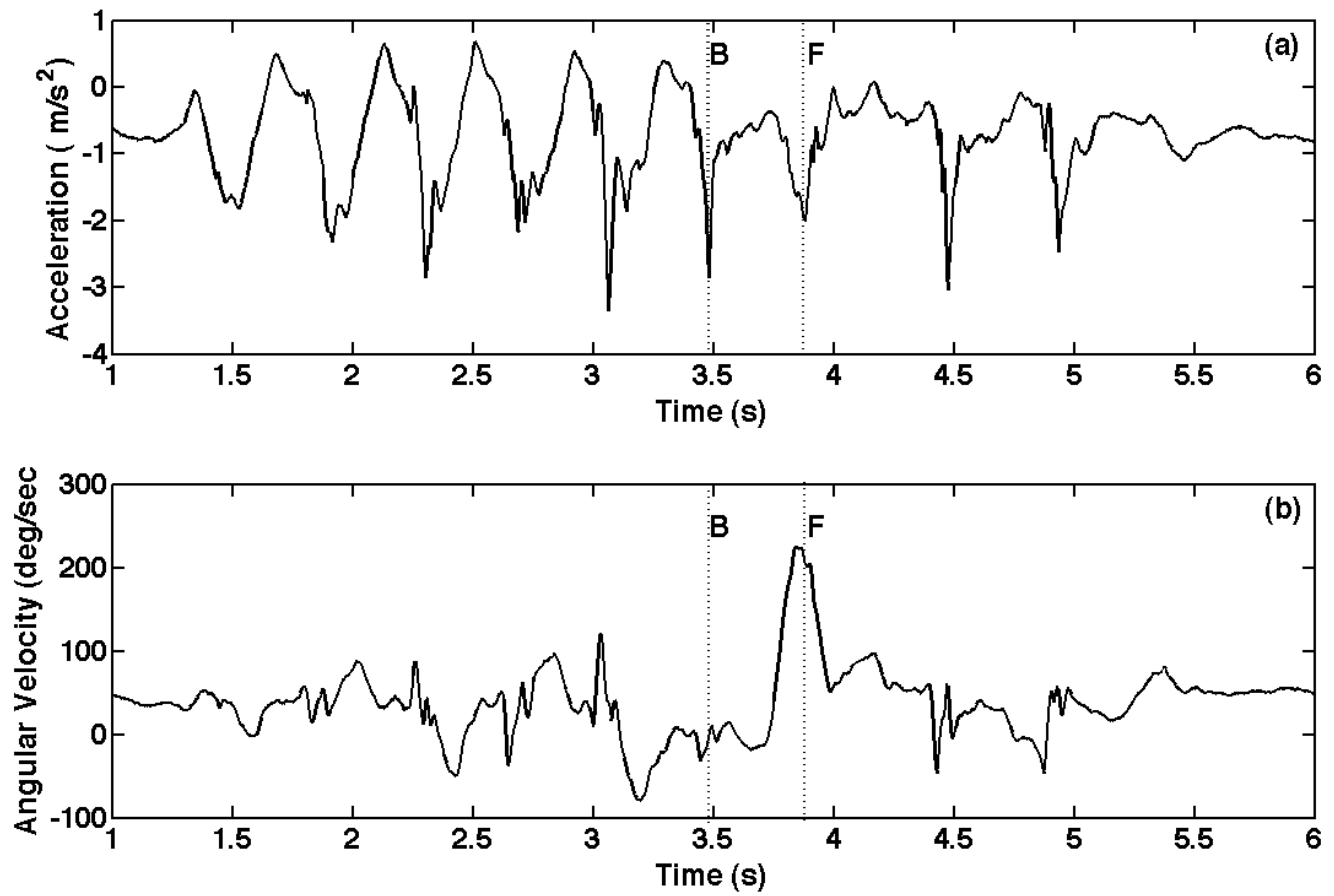

Figure 2 


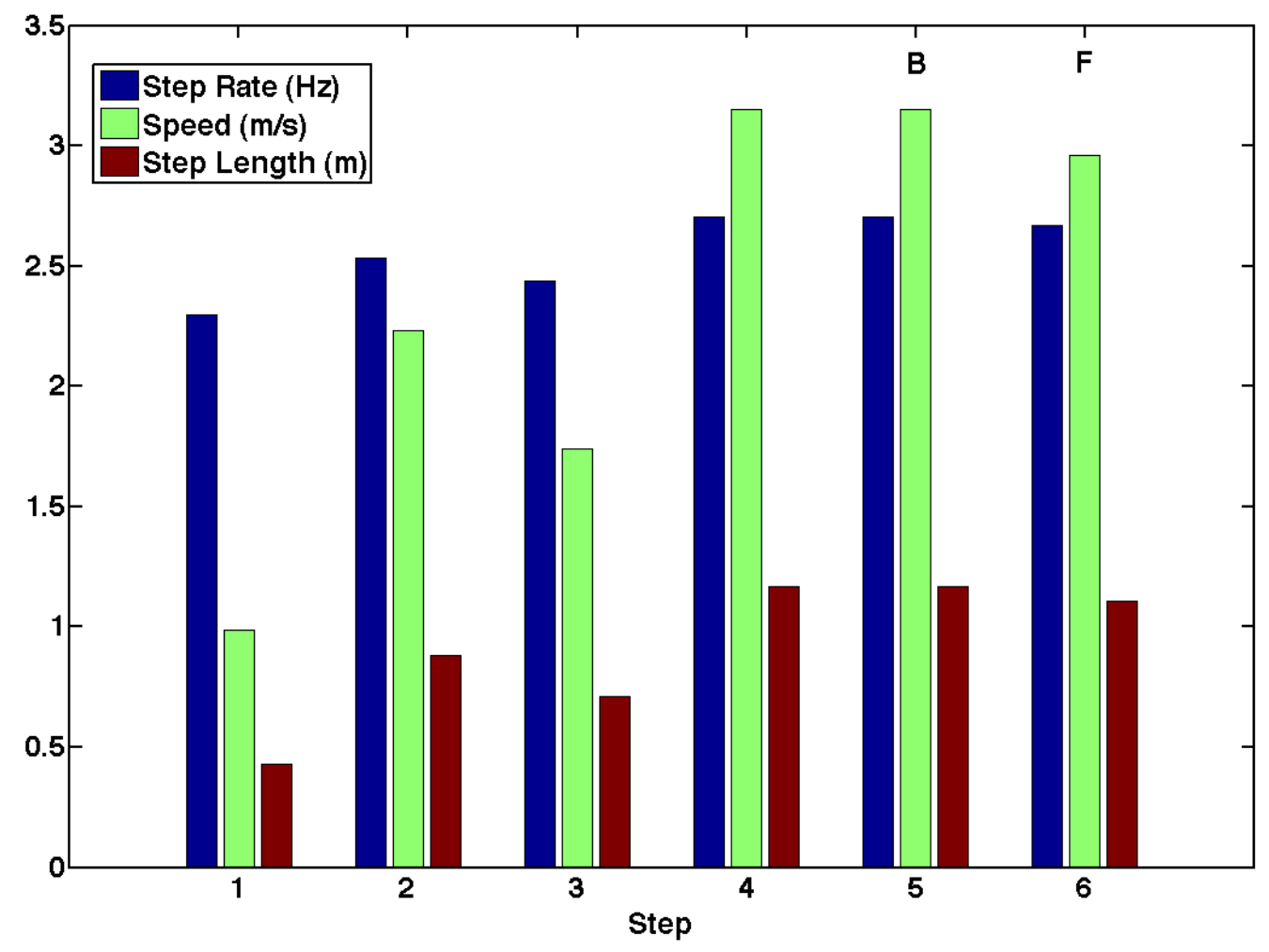

Figure 3 